\title{
Exploring the roles of fecundity-related long non-coding RNAs and mRNAs in the adrenal glands of small-tailed Han Sheep
}

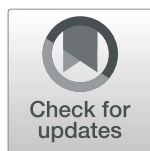

Qing Xia ${ }^{1}$, Qiuling Li ${ }^{2}$, Shangquan $\mathrm{Gan}^{3}$, Xiaofei Guo ${ }^{4}$, Xiaosheng Zhang ${ }^{4}$, Jinlong Zhang ${ }^{4}$ and Mingxing $\mathrm{Chu}^{{ }^{*}}$

\begin{abstract}
Background: Long non-coding RNAs (IncRNAs) can play important roles in uterine and ovarian functions. However, little researches have been done on the role of IncRNAs in the adrenal gland of sheep. Herein, RNA sequencing was used to compare and analyze gene expressions in adrenal tissues between follicular phases and luteal phases in $F e c B^{B B}(\mathrm{MM})$ and $\mathrm{FeCB}^{++}(\mathrm{WW})$ sheep, respectively, and differentially expressed IncRNAs and genes associated with reproduction were identified.

Results: In MM sheep, 38 IncRNAs and 545 mRNAs were differentially expressed in the adrenal gland between the luteal and follicular phases; In WW sheep, 513 differentially expressed IncRNAs and 2481 mRNAs were identified. Gene Ontology and Kyoto Encyclopedia of Genes and Genomes enrichment analyses indicated that differentially expressed IncRNAs and their target genes are mainly involved in the circadian rhythm, the mitogen activated protein kinase, thyroid, ovarian steroidogenesis and transforming growth factor beta signaling pathways. Differentially expressed IncRNAs can regulate reproduction by modulating genes involved in these signaling pathways and biological processes. Specifically, XLOC_254761, XLOC_357966, 105,614,839 and XLOC_212877 targeting CREB1, PER3, SMAD1 and TGFBR2, respectively, appear to play key regulatory roles.
\end{abstract}

Conclusion: These results broaden our understanding of IncRNAs in adrenal gland of sheep and provide new insights into the molecular mechanisms underlying sheep reproduction.

Keywords: LncRNAs, Follicle phase, Luteal phase, Small-tailed Han sheep, Adrenal

\section{Background}

Small-tailed Han sheep are famous for their high fertility, precocious puberty, good fur quality and tall body shape in China [1]. The behavior of estrus and mate in Small-tailed Han sheep appear year-round. The lambing rate of primiparous ewes is about $200 \%$, and in produced ewes is higher than $250 \%$ [1]. $\mathrm{Fec}^{B B}$ mutations have huge economic benefits in production, which can significantly increase the number of ovulation and lambs in sheep.

\footnotetext{
* Correspondence: mxchu@263.net

${ }^{1}$ Key Laboratory of Animal Genetics and Breeding and Reproduction of the Ministry of Agriculture and Rural Affairs, Institute of Animal Science, Chinese Academy of Agricultural Sciences, Beijing 100193, P. R. China

Full list of author information is available at the end of the article
}

According to the previous research report of this team, all three genotypes of $\mathrm{FecB}\left(\mathrm{FecB} \mathrm{BB}^{\mathrm{B}}, \mathrm{FecB}^{\mathrm{B+}}\right.$ and $\left.\mathrm{FecB}^{++}\right)$ are distributed in Small-tailed Han sheep, and there is a significant correlation between the three genotypes of $F e c B$ and the lamb size of ewes [2]. Therefore, Smalltailed Han sheep can be used as an ideal animal model to study the molecular mechanism of $F e c B$ gene regulation of reproductive traits.

In recent years, there have arisen many methods for identifying differentially expressed candidate long noncoding RNAs (lncRNAs) and genes using transcriptome sequencing. lncRNAs are RNAs of $>200$ nucleotides in length. Studies have shown that lncRNAs play important roles in many life activities, such as dose-compensation

C The Author(s). 2020 Open Access This article is licensed under a Creative Commons Attribution 4.0 International License, which permits use, sharing, adaptation, distribution and reproduction in any medium or format, as long as you give appropriate credit to the original author(s) and the source, provide a link to the Creative Commons licence, and indicate if changes were made. The images or other third party material in this article are included in the article's Creative Commons licence, unless indicated otherwise in a credit line to the material. If material is not included in the article's Creative Commons licence and your intended use is not permitted by statutory regulation or exceeds the permitted use, you will need to obtain permission directly from the copyright holder. To view a copy of this licence, visit http://creativecommons.org/licenses/by/4.0/ The Creative Commons Public Domain Dedication waiver (http://creativecommons.org/publicdomain/zero/1.0/) applies to the data made available in this article, unless otherwise stated in a credit line to the data. 
effects, epigenetic regulation, cell cycle regulation, cell differentiation and regulation, and have become a hot research topic in genetics [3-6]. For example, Yang et al. (2018) identified differentially expressed lncRNAs and mRNAs in the testes of prepubertal and mature rams that were enriched in spermatogenetic and male gonadal developmental signaling pathways [5]. Zheng (2019) analyzed the pituitaries of $\mathrm{Hu}$ sheep with high and low fertility and found 57 differentially expressed lncRNAs and 298 differentially expressed mRNAs [6]. Miao et al. (2016, 2017) analyzed the ovaries of Small-tailed Han and Dorset sheep strains and found that differentially expressed lncRNAs were significantly enriched in the oxytocin signaling pathway. Methylation of lncRNAs might contribute to improving the reproduction of Small-tailed Han sheep [3, 4]. Feng (2018) identified 76 differentially expressed mRNAs and 5 differentially expressed lncRNAs by analyzing the ovaries of $\mathrm{Hu}$ sheep with high and low reproduction rates [7]. These studies showed that lncRNAs in the pituitary and ovaries of sheep have regulatory functions in reproduction. It is known that the sheep adrenal gland also has an impact on reproduction [8-12], but studies on the functions of IncRNAs in this organ are limited.

In this study, the differentially expressed genes in the adrenal gland between Small-tailed Han sheep with $\mathrm{FeCB}^{B B}$ (MM) and Small-tailed Han sheep with $\mathrm{FecB}^{++}$ (WW) (hitherto simply MM and WW sheep) were analyzed using RNA sequencing (RNA-Seq). The molecular mechanisms of differentially expressed lncRNAs and genes in the adrenal gland related to reproduction were subjected to a preliminary exploration. Our results provide an effective theoretical basis for studying the molecular mechanisms by which lncRNAs regulate sheep reproduction.

\section{Results}

Transcript assembly and quality control

The RNA-seq data of 12 samples were subjected to quality control, and the results are shown in Table 1 . The clean reads for each sample ranged from 80 to 100 million and the Q30 values ranged from 92.50 to $95.30 \%$. About $89.47-91.63 \%$ of the clean reads were mapped to the sheep reference genome, and about $80 \%$ were mapped uniquely.

\section{LncRNA identification and characterization}

A total of 17,201 candidate lncRNAs was identified, including 1174 anti-sense lncRNAs, 10,636 intronic lncRNAs and 5391 large intergenic (linc) RNAs (Fig. 1a). As shown in Fig. 1b, most lncRNAs had two exons: significantly fewer than the exons of mRNAs. The expression levels of mRNAs and lncRNAs were further analyzed according to FPKM values, and the boxplot (Fig. 1c) shows that the expression levels of mRNAs in the adrenal tissues were higher than lncRNAs. The distributions of lncRNAs and mRNAs lengths were consistent (Fig. 1d).

\section{Expression levels of genes and differentially expressed analysis}

The Fig. 1c box plot shows that lncRNAs transcript expression levels were all lower than those of mRNAs in adrenal of both MM and WW Small-tailed Han sheep. Based on $P$ values $<0.05$, differentially expressed gene analysis of adrenal tissues showed that there were 15 lncRNAs upregulated and 23 downregulated in MM sheep; 279 mRNAs were upregulated and 266 downregulated in MM sheep (Supplemental Table S1). In WW sheep, 354 lncRNAs were upregulated and 159

Table 1 Summary of quality data after quality control

\begin{tabular}{|c|c|c|c|c|c|c|}
\hline Sample & Raw read s & Clean read s & Clean reads rate(\%) & Q30(\%) & Total mapped rea ds & Uniquely mapped rea ds \\
\hline$\overline{M F 1}$ & $121,899,310$ & $118,357,160$ & 97.09 & 95.30 & $90.83 \%$ & $82.16 \%$ \\
\hline MF2 & $100,514,168$ & $97,878,852$ & 97.38 & 94.12 & $91.63 \%$ & $83.62 \%$ \\
\hline MF3 & $93,565,822$ & $91,784,982$ & 98.10 & 94.30 & $90.28 \%$ & $84.74 \%$ \\
\hline MF1 & $89,881,752$ & $86,058,280$ & 95.75 & 92.68 & $89.61 \%$ & $85.02 \%$ \\
\hline MF2 & $91,763,718$ & $88,508,870$ & 96.45 & 92.79 & $90.52 \%$ & $82.55 \%$ \\
\hline MF3 & $88,087,492$ & $84,832,940$ & 96.31 & 92.77 & $89.98 \%$ & $82.94 \%$ \\
\hline MF1 & $109,076,604$ & $105,978,806$ & 97.16 & 93.94 & $89.76 \%$ & $81.41 \%$ \\
\hline MF2 & $106,524,486$ & $101,591,104$ & 95.37 & 93.01 & $89.47 \%$ & $80.75 \%$ \\
\hline MF3 & $89,866,984$ & $86,416,914$ & 96.16 & 92.50 & $89.87 \%$ & $81.97 \%$ \\
\hline MF1 & $87,611,256$ & $85,783,998$ & 97.91 & 92.96 & $91.46 \%$ & $81.32 \%$ \\
\hline MF2 & $102,807,796$ & $99,286,834$ & 96.58 & 93.28 & $89.95 \%$ & $80.54 \%$ \\
\hline MF3 & $102,733,338$ & $99,271,932$ & 96.63 & 92.65 & $90.76 \%$ & $82.68 \%$ \\
\hline
\end{tabular}

Note: MF and ML represent follicular phase and luteal phase in MM sheep, respectively; WF and WL represent follicular phase and luteal phase in WW sheep, respectively 


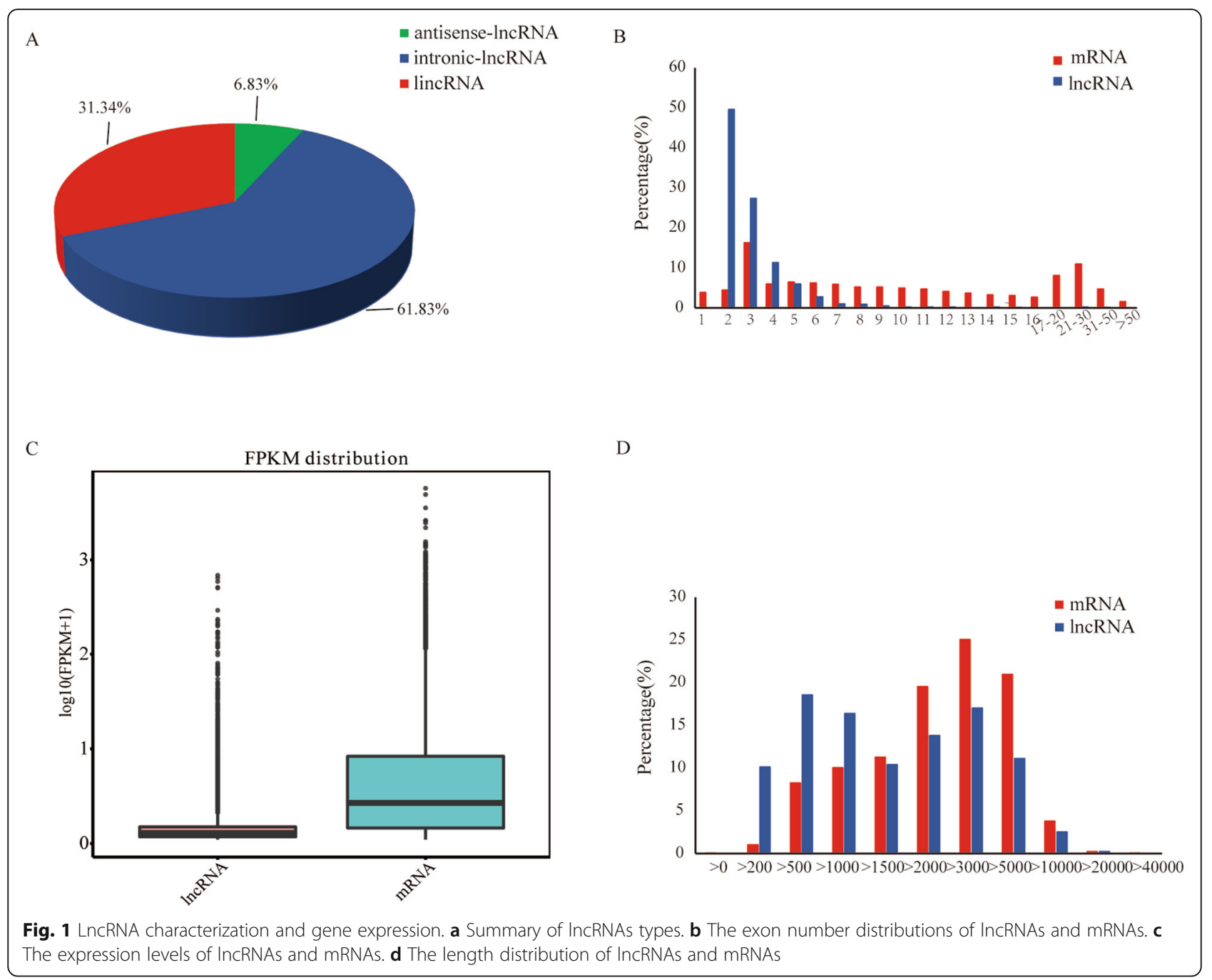

downregulated; 1334 mRNAs were upregulated and 1147 downregulated (Supplemental Table S1 \& S2).

\section{GO annotation and KEGG enrichment analysis of differentially expressed mRNAs}

The GO enrichment analysis of all differentially expressed mRNAs showed that they were categorized into BP, CC, and MF. The differentially expressed mRNAs in MM and WW sheep associated with reproduction were mainly annotated in biological processes, and these were involved in reproduction processes, mating behavior, ovarian follicle cell development, the mitogen-activated protein kinase (MAPK) cascade and steroid metabolic processes in MM sheep (Fig. 2a, Supplemental Table S3). In WW sheep, these were involved in the Wnt receptor signaling pathway, meiosis, post-embryonic development, asexual reproduction and responses to steroid hormone stimuli (Fig. 2b, Supplemental Table S4).
The KEGG enrichment analysis showed that the majority of differentially expressed mRNAs were enriched in the same pathways in both the MM and WW sheep. The differentially expressed mRNAs were enriched in progesteronemediated oocyte maturation, the $M A P K$ signaling pathway, circadian rhythm, oocyte meiosis and insulin signaling pathway (Fig. 2c and d, Supplemental Table S5).

\section{GO annotation and KEGG enrichment analysis of target genes of IncRNAs}

LncRNAs target genes were analyzed according to GO annotation and KEGG enrichment. As shown by the GO annotation results, lncRNA target genes associated with reproduction were also mainly annotated in biological processes in the MM and WW sheep. As shown in Fig. 3, both cis-target genes and trans-target genes associated with reproduction were enriched in biological processes in the MM type and WW sheep. In the MM sheep, cistargets were mainly enriched for female gamete generation, reproduction and oogenesis, and trans-targets 


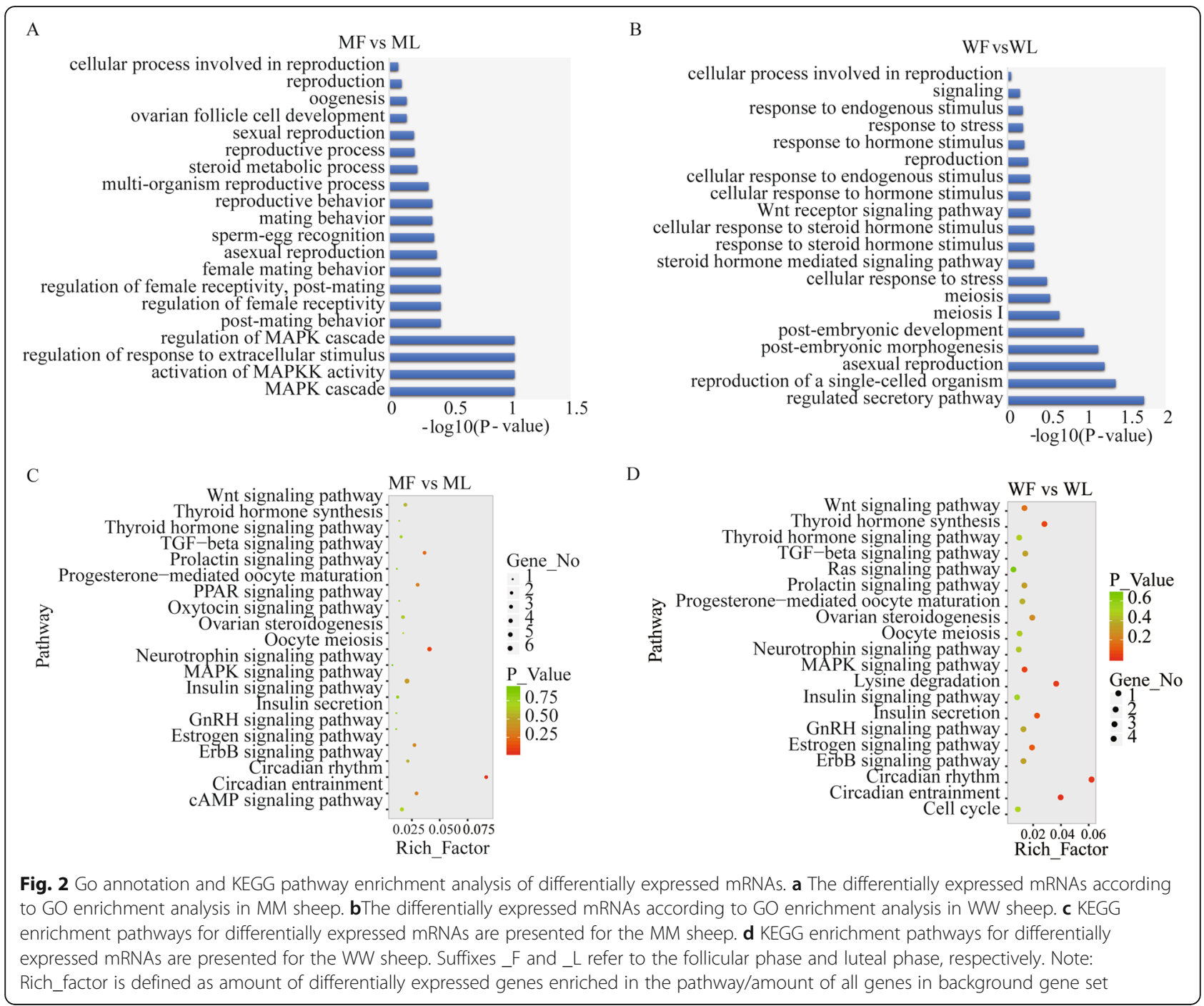

were enriched for the Wnt receptor signaling pathway, reproduction and circadian rhythm (Fig. 3a). Cis-targets and trans-targets were all mainly enriched for reproduction, gamete generation and ovarian follicle cell development (Fig. 3b).

The KEGG enrichment analysis associated with reproduction showed that the majority of cis-target genes and trans-target genes were enriched in the same pathways in MM and WW sheep (Fig. 3). In MM sheep, the lncRNA targets were assigned to 20 reproduction pathways, such as Wnt signaling pathway, transforming growth factor beta $(T G F \beta)$ signaling pathway, ovarian steroidogenesis, $M A P K$ signaling pathway, circadian rhythm and other (Fig. 3c). The lncRNA targets were enriched in progesterone-mediated oocyte maturation, $M A P K$ signaling pathway, circadian rhythm, oocyte meiosis and insulin signaling pathway (Fig. 3d; Supplementary Table S6).

\section{LncRNAs and mRNAs co-expression network analysis}

In the MM sheep, a lncRNA/mRNA co-expression network was constructed using 46 differentially expressed lncRNAs and 17 target genes involved in reproductive-related pathways. As shown in Fig. 4, some lncRNA targets are located in the center of the network, for example BAD, PLCB3, MYL6, MEL6B, CACNA2S, CSNK1A1, PRKG1 and HIF1A. In the WW sheep, a lncRNA-mRNA co-expression network was constructed for reproductive-related pathways using 29 differentially expressed lncRNAs and 12 target genes. As shown in Fig. 5, some lncRNA targets are located in the center of the network, for example MAP 3 K11, ANAPC11, FKBP5, FLNB and PDPK1. The network model shows that reproductive-related lncRNA targets are co-expressed with lncRNAs, indicating that IncRNAs and mRNAs are mutually regulated during reproduction. 


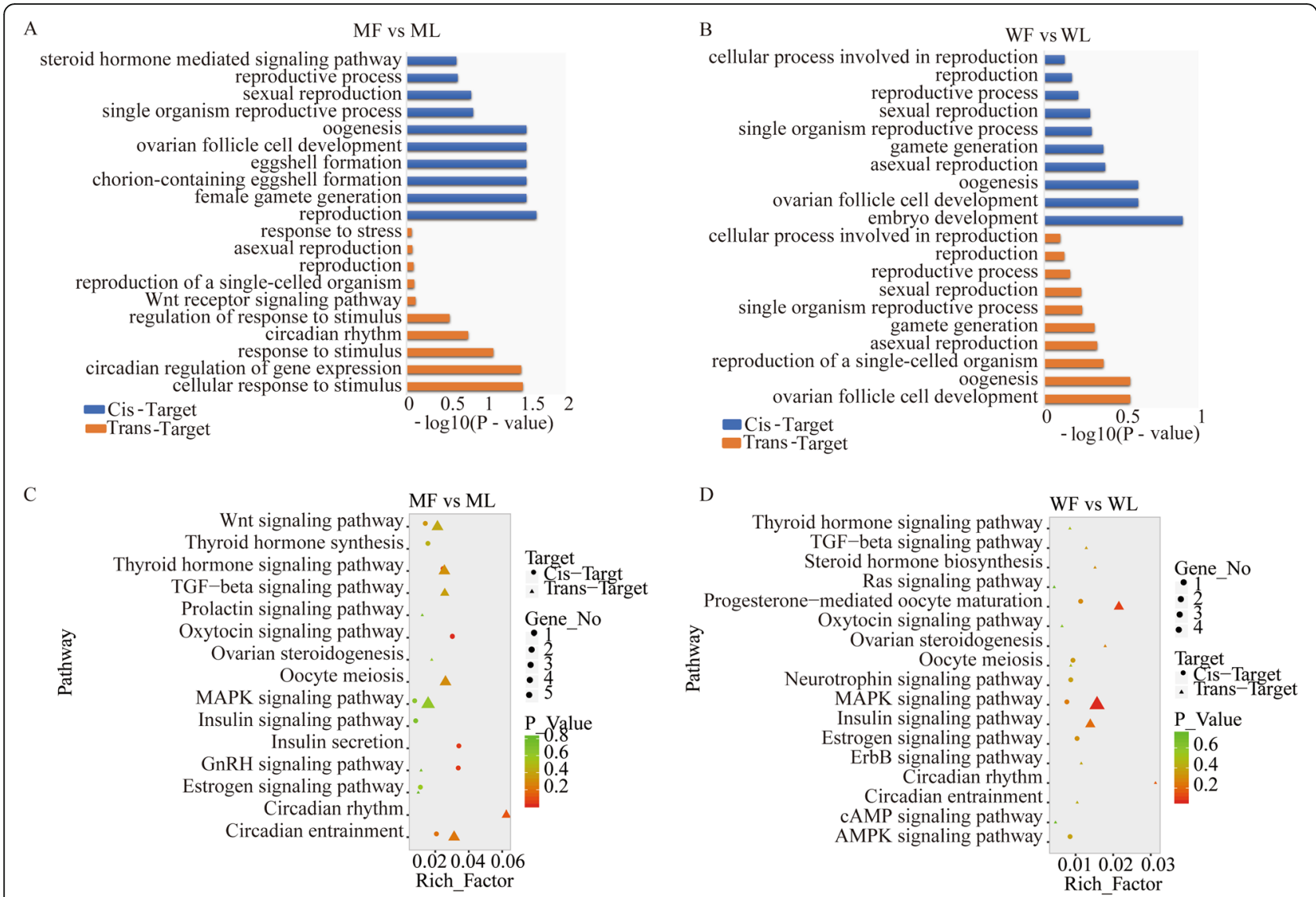

Fig. $3 \mathrm{GO}$ annotation and KEGG pathway analysis of differentially expressed IncRNA targets. aThe differentially expressed IncRNA target gene GO enrichment analysis for MM sheep. bThe differentially expressed IncRNA target gene GO enrichment analysis for WW sheep. c KEGG enrichment pathways for differentially expressed IncRNA targets are presented for MM sheep. d KEGG enrichment pathways for differentially expressed IncRNA targets are presented for WW sheep. Suffixes_F and_L refer to the follicular phase and luteal phase, respectively. Note: Rich_factor is defined as amount of differentially expressed genes enriched in the pathway/amount of all genes in background gene set

\section{RNA-Seq data validation by $\mathrm{qPCR}$}

To further validate the sequencing data, 8 differentially expressed lncRNAs and 7 differentially expressed lncRNA target genes were selected to detect expression levels by RT-qPCR (Supplementary Table S7). As shown in Fig. 6, BAD, XLOC_397965, XLOC_2882304, XLOC_ 212066 and $X L O C \_108057$ were upregulated in the adrenal glands of the MM sheep and PER3, SMAD1, TGFBR2 were downregulated in MM sheep. NTRK2, SMAD1, CREB1, XLOC_274809, XLOC_374827, XLOC_ 381122 and XLOC_393803 were upregulated in the WW sheep. These results were consistent with the transcriptome data results.

\section{Discussion}

It is known that lncRNAs play an important regulatory role in sheep reproduction. Functional lncRNAs have been identified in the brain, heart, kidney, liver, lung, ovary, skin, white adipose tissue, and pituitary in sheep [13]. In addition, they have also been identified in human, mouse and pig uterine tissues $[14,15]$. The adrenal gland, hypothalamus and pituitary were concluded in the hypothalamic-pituitary-adrenal axis (HPA), and the HPA axis interacts with the hypothalamic-pituitarygonadal (HPG) axis at the brain and pituitary levels to maintain a balance between sheep reproduction and survival. However, little research has been done on lncRNAs and their targets in sheep adrenal glands.

GO annotation and KEGG enrichment analysis indicated that the differentially expressed lncRNA targets were mainly involved in the Wnt signaling pathway, TGF- $\beta$ signaling pathway, ovarian steroidogenesis, MAPK signaling pathway and circadian rhythm. Analysis of differential lncRNA-mRNA co-expression patterns and functions of target genes revealed that lncRNA might affect the fecundity of sheep by modulating genes associated with the above signaling pathways and biological processes. Among the MM sheep, these pathways were enriched for four differentially expressed lncRNAs (XLOC_212877, XLOC_357966, 105,614,839, XLOC_ 

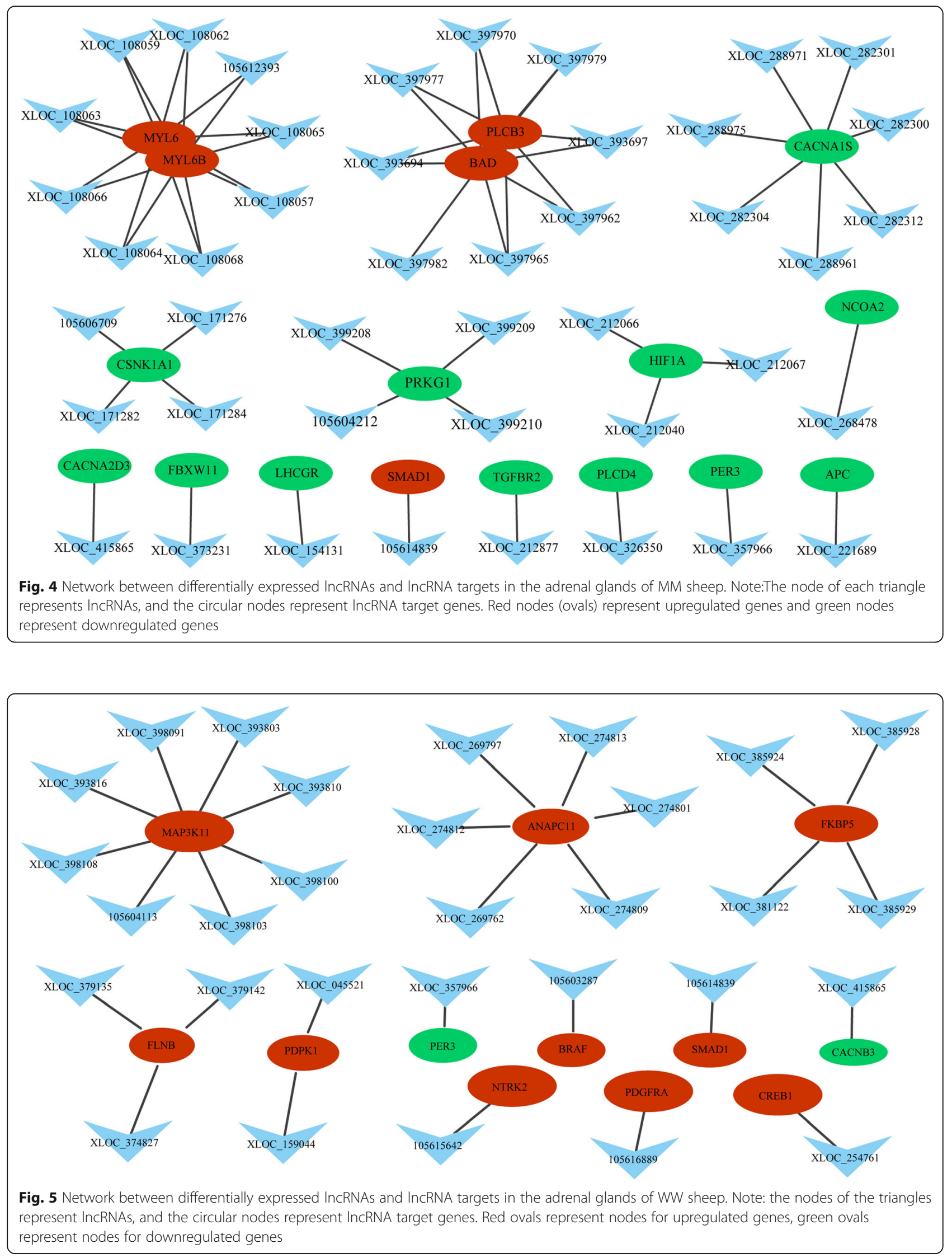

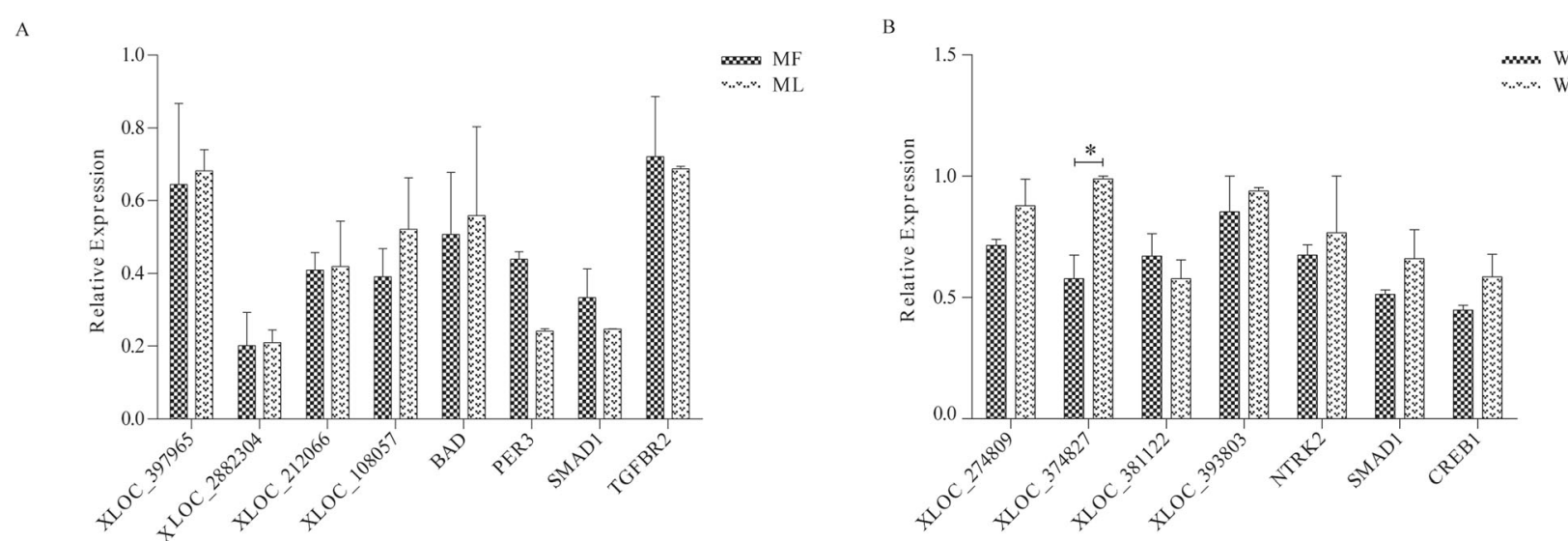

Fig. 6 RT-qPCR verification of differentially expressed genes. a Verification of the differentially expressed genes in MM sheep. $\mathbf{b}$ Verification of the differentially expressed genes in WW sheep. Suffixes_F and _L refer to the follicular and luteal phases, respectively

154131) and four lncRNA targets (TGFBR2, PER3, SMAD1, LHCGR). In the WW sheep, these pathways were enriched in three differentially expressed lncRNAs (XLOC_254761, 105,603,287, 105,615,642) and 3 lncRNA targets (CREB1, BRAF, NTRK2).

The HPA axis can be regulated by stress hormone signaling. The paraventricular nucleus in the hypothalamus is activated to release corticotropin releasing hormone $(\mathrm{CRH})$, and $\mathrm{CRH}$ stimulates the release of adrenocorticotropic hormone (ACTH). ACTH stimulates the adrenal gland to release glucocorticoids $[16,17]$. The HPA can interact with the HPG. For example, estradiol secreted by the ovaries can enhance the activity of the HPA axis. At the level of hypothalamus, CRH inhibits the secretion of gonadotropin releasing hormone $(\mathrm{GnRH})$. In the pituitary, luteinizing hormone levels were significantly reduced in mice chronically exposed to corticosterone [18]. At the level of the adrenal gland, women with congenital adrenal hyperplasia can suffer from impaired fertility or impaired steroid secretion caused by increased androgen levels [19]. High concentrations of glucocorticoids have an inhibitory effect on the activities of GnRH-secreting neurons, pituitary gonadotropins and gonads [12]. In addition, glucocorticoids inhibit thyroid stimulating hormone secretion and reduce the conversion of $\mathrm{T} 3$ (inactive thyroxine) to $\mathrm{T} 4$ (effective triiodothyronine) during stress. Endogenous thyrotropin-releasing hormone and TSH secretion levels can be inhibited by CRH-induced somatostatin [9]. In sheep, acute stress inhibits the release of LH from the pituitary by inhibiting the synthesis of $\mathrm{GnRH}$ and $\mathrm{GnRH}$ receptors and promotes functional enhancement of gonadotropin-inhibiting hormone-secreting neurons [20-22]. Clearly, the adrenal gland plays an important role in the HPG.

Cyclic AMP response element binding protein 1 (encoded by CREB1) is a member of the CREB family, which is present in different ovarian compartments, including follicular granulosa cells [23-25]. CREB protein concentration increases during sexual maturation and ovarian follicular development [26, 27]. CREB1 can bind to the estrogen receptor alpha to play a key role in chicken sexual maturation [28]. The gene encoding serine/threonine kinase $(B R A F)$ is a proto-oncogene that has been identified previously as a candidate target gene in human endometriosis. CREB1 is a potential transcription factor of $B R A F$. Compared with normal endometrial tissue, the mRNA expression levels of $B R A F$ and CREB1 are significantly upregulated in endometrial tissues of patients with endometriosis; thus, CREB1 can increase the expression of $B R A F$ and regulate cell proliferation by binding directly to $B R A F$ in such women [29]. One study determined the role of CREB1 in mouse granulocytes (mGCs) by knocking down the expression of CREB1. It was found that CREB1 could be regulated by steroid synthesis, cell proliferation, cell cycle, apoptosis and other follicular factors as key regulators of mGCs [30]. Moreover, XLOC_254761 can transregulate CREB1, and 10,563,287 trans-regulated BRAF. CREB1 and BRAF are enriched in progesterone-mediated oocyte maturation. These results indicated that those genes enriched in progesterone-mediated oocyte maturation may promote follicular maturation and regulate reproduction in Small-tailed Han sheep.

Using six estrous Hu sheep ovaries and six nonestrous China merino sheep ovaries to identify genes associated with off-season reproduction, the gene encoding neurotrophic receptor tyrosine kinase 2 (NTRK2) was found to be differentially upregulated in the ovaries [31]. The NTRK2 gene encodes the NTRK2 receptor for neurotrophin-4/5 and brain-derived neurotrophic factor leading to oocyte death in late adolescence, loss of follicular tissue and infertility in early adulthood. The preovulatory gonadotropin surge promotes oocyte survival 
at the onset of reproductive cyclicity by inducing oocyte expression of NTRK2 in Hu sheep [32]. 105,615,642 can transregulate NTRK2, which is enriched in the MAPK signaling pathway. The results suggest that NTRK2 might regulate follicular survival.

The expression level of the gene encoding luteinizing hormone receptor $(L H C G R)$ is low in mural granulosa cells and cumulus cells of antral follicles, and LHCGR is induced in granulosa cells by follicle stimulating hormone (FSH). The synthesis of LHCGR is mediated by retinoic acid (RA), the demethylation of its promoter region is a key mechanism regulating cell type-specific differentiation during follicular development [33].

Mothers against decapentaplegic homolog 1 (SMAD1) is a downstream signal transduction element of bone morphogenetic protein [34, 35], and SMAD1 responds to bone morphogenetic protein 15 (BMP15), which is important for proliferation of granulosa cells in sheep [36]. The transforming growth factor-beta (TGF $\beta$ ) subfamily is encoded by TGFB1, TGFB2 and TGFB3. In mammals, there are seven TGF-1 receptor subtypes (encoded by TGFBR1) and five type 2 receptor subtypes (TGFBR2) associated with signal transduction ligand of the TGF $\beta$ superfamily $[37,38]$. The TGF $\beta$ superfamily ligand initiates an intracellular signaling pathway upon binding to a cell surface receptor complex. In addition, $T G F B R 2$ in the TGF- $\beta$ signaling pathway were found to be associated with litter size in pigs [39]. There was a significant correlation between litter size in mutant indigenous Chinese $\mathrm{Hu}$ sheep and mutations in the three genes encoding the TGF $\beta$ superfamily $(F e c B, G D F 9$, and TGFBR2); moreover, the $F e c B, G D F 9$ and TGFBR2 polymorphisms are considered to be potentially important genetic markers in marker-assisted selection (MAS) strategies to increase litter size in these sheep) [40]. Moreover, here we show that 105,614,839 and XLOC_ 212877 cis-regulate $S M A D 1$ and TGFBR2, respectively. These genes are enriched in the TGF- $\beta$ signaling pathway. The results indicate that SMAD1 and TGFBR2 might regulate reproduction in terms of uterine decidual function and litter size.

\section{Conclusion}

In summary, the adrenal gland plays a key role in sheep female reproductive processes. For example, the adrenal gland affects the reproductive of sheep through hypothalamic-pituitary-adrenal axis. These functions of the adrenal gland are achieved by regulating different signaling pathways and related genes. In this study, we screened the differentially expressed lncRNAs (XLOC_ 212877, XLOC_357966, XLOC_154131, XLOC_254761, $105,614,839,105,603,287$ and 105,615,642) and differentially expressed lncRNA target genes (TGFBR2, PER3, SMAD1, LHCGR, CREB1, BRAF and NTRK2) associated with sheep prolificacy, and constructed networks of interactions between lncRNAs and mRNAs. The lncRNA and mRNAs associated with sheep breeding were enriched by KEGG analysis. The results of the study will help to elucidate the regulatory mechanisms of lncRNAs in sheep reproduction.

\section{Methods}

\section{Ethics statement}

All the animals were authorized by the Science Research Department (in charge of animal welfare issue) of the Institute of Animal Sciences, Chinese Academy of Agricultural Sciences (IAS-CAAS; Beijing, China). In addition, ethical approval of animal survival was given by the animal ethics committee of IAS-CAAS (No. IAAS 2019-449, 18 September 2019).

\section{Animals and sample collection}

Animals were from the core group of Small-tailed Han sheep in the Luxi area of Shandong Province, P. R. China. All were free to eat and drink. We chose healthy nonpregnant sheep aged 2-4 years. Jugular vein blood was collected and the $F e c B$ mutation was identified by TaqMan assays. A total of 12 sheep (six WW and six $\mathrm{MM}$, respectively) were used for the experiments. All experiments complied with the rules established by the animal ethics committee of IAS-CAAS (No. IAAS 2019449, 18 September 2019).

All sheep were treated with vaginal sponges (InterAg Co., Ltd., New Zealand) (progesterone $300 \mathrm{mg}$ ) for 12 days to synchronize estrus. Three WWW and three MM ewes were euthanized (Intravenous pentobarbital (100 $\mathrm{mg} / \mathrm{kg}$ ))on the 50th hours after removing the vaginal sponges, and the adrenal glands were collected (follicular phase; WF and MF, respectively), and of the remaining six sheep (three in each group) were euthanized (Intravenous pentobarbital $(100 \mathrm{mg} / \mathrm{kg}))$ on the 7 th day after sponge removal (luteal phase; WL and ML, respectively) and the adrenal glands were collected. All samples were stored immediately at $-80^{\circ} \mathrm{C}$ for total RNA extraction. All experiments complied with the rules established by the the animal ethics committee of IAS-CAAS (No. IAAS 2019-449, 18 September 2019).

\section{RNA isolation, library preparation and sequencing}

Total RNA was extracted from the adrenal glands of all 12 ewes using TRIzol (Thermo Fisher Scientific, Waltham, MA, USA) in accordance with the manufacturer's instruction. RNA purity was checked using a Nano Photometer ${ }^{\circ}$ spectrophotometer (IMPLEN, Westlake Village, CA, USA). RNA concentrations were measured using Qubit $^{\circ}$ RNA Assay kits in a Qubit $^{\circ} 2.0$ Fluorometer (Thermo Fisher Scientific, Waltham, MA, USA). RNA integrity was assessed using the RNA Nano 6000 Assay 
kits of the Bioanalyzer 2100 system (Agilent Technologies, Santa Clara, CA, USA).

The lncRNA library we generated was chain-specific. The method of reverse transcription synthesis of the first strand of cDNA is the same as the New England Biolabs (NEB) general library construction (NEB, Ipswich, MA, USA). The difference is that when the second strand is synthesized, the dTTP in the dNTPs is replaced by dUTP, followed by cDNA end repair, addition of an A tail, ligation sequencing and length screening. Then we used the USER enzyme (New England Biolabs, Inc., Ipswich, MA, USA) to degrade the second strand of Ucontaining cDNA and performed polymerase chain reaction (PCR) amplification to obtain a library. After the IncRNA library was established, the library preparations were sequenced on an Illumina Hiseq platform (Illumina, San Diego, CA, USA). Raw data of the performed RNA-seq have been recorded in the SRA public database (Accession number: SRP222893).

Reference genome mapping and transcriptome assembly Raw reads were obtained by removing reads with an adapter, reads containing $>10 \%$ poly- $\mathrm{N}$ sequences and low-quality reads. At the same time, Q20, Q30 and GC contents of the clean data were calculated. An index of the reference genome was built using bowtie 2 v. 2.2.8 (http://bowtie-bio.sourceforge.net/bowtie2/index.shtml) [41] and paired-end clean reads were aligned to the reference genome Oar_v3.1 using HISAT2 v. 2.0.4 (https:// ccb.jhu.edu/software/hisat2/index.shtml) [42].

The mapped reads of each sample were assembled using StringTie v. 1.3.1 (https://ccb.jhu.edu/software/ stringtie/) [42] in a reference-based approach. StringTie uses a novel network flow algorithm as well as an optional de novo assembly step to assemble and quantitate full-length transcripts representing multiple splice variants for each gene locus.

\section{Identification of potential IncRNA candidates}

Potential lncRNA candidates were identified as follows: selecting transcripts with exon number $\geq 2$ and transcript length $>200 \mathrm{nt}$. The transcripts obtained by combining each sample using Cuffdiff v. 2.1.1 (https:// software.broadinstitute.org/cancer/software/genepattern/ modules/docs/Cuffcompare/8) [43], and transcripts with uncertain chain direction were removed to obtain complete transcriptome information.

The intersection of transcripts with no coding potential in these software analysis results was used as the lncRNA data set. CNCI v. 2.0 (https://github.com/wwwbioinfo-org/CNCI) [44], CPC v. 2.81 (http://cpc.cbi.pku. edu.cn/) [45] and PFAM v. 1.3 (https://pfam.xfam.org) [46] were used to evaluate the protein coding potential of the transcript and the results of these software as the end result.

\section{Analysis of differentially expressed genes}

Gene expression estimation using the FPKM value expected number of Fragments Per Kilobase of transcript sequence per million base pairs sequenced) eliminates the effect of sequencing depth and gene length on fragment counts. Different types of transcripts (lncRNAs and mRNAs) were analyzed for differences using Cuffdiff v. 2.1.1 (https://software.broadinstitute.org/cancer/software/ genepattern/modules/docs/Cuffdiff/7) [43]. LncRNAs and mRNAs with $P$ values $<0.05$ were considered as differentially expression between the two groups of data.

\section{GO annotation and KEGG pathway enrichment analysis of differentially expressed genes}

The GO is an international standard system including molecular functions, biological processes, and cellular components for classifying gene function. Pathway enrichment analysis can identify major metabolic pathways and signaling pathways enriched by differentially expressed genes. Enrichment analysis was performed on each Pathway in KEGG using a hypergeometric test. The calculated $P$ value and 0.05 being defined as the significant threshold, the genes were screened and enriched for the pathways. Next, the significance of the pathway enrichment analysis was corrected by FDR, and the corrected $P$-value (Q-value) was obtained. Differentially expressed genes were further studied using the GO and KEGG databases to study the functions of the genes and identify the pathways in which they participate. If a $\mathrm{P}$ value was $\leq 0.05$, enrichment was considered significant.

GO annotation and KEGG pathway enrichment analysis of differentially expressed IncRNA targets

Differentially expressed lncRNAs regulate the target genes by cis-regulating nearby genes and transregulating distal protein-coding genes. Here, proteincoding genes with a distance of less than $100 \mathrm{~Kb}$ were assumed to be the cis-target genes, and Pearson correlation coefficients with the lncRNAs of $>0.95$ were assumed to represent the trans-target genes [47]. Statistical enrichment of differentially expressed lncRNA target genes in the GO annotation and KEGG pathways were evaluated, and $P$ value $\leq 0.05$ was considered significant enrichment.

\section{Construction of IncRNA/mRNA networks}

To infer the functions of differentially expressed lncRNAs and their target genes in sheep prolificacy, we constructed a network based on mRNAs and lncRNAs in Cytoscape v. 3.1.1 (https://cytoscape.org). 


\section{Reverse transcription (RT)-qPCR verification}

RT-qPCR was used to verify the expression levels of differentially expressed lncRNAs and their targets. About $0.1 \mu \mathrm{g}$ of RNA was used per sample and this was reverse transcribed into cDNA using RT reagents (Thermo Fisher Scientific, Waltham, MA, USA). All experiments were performed in triplicate, and $\beta$-actin was used as an internal reference to normalize target gene expression. The qPCR was performed on a LightCycler 480II (Roche, Basel, Sweden) using SYBR Premix Ex Taq II. The procedure involved 40 cycles of pre-denaturation at $95^{\circ} \mathrm{C}$ for $5 \mathrm{~s}$; denaturation at $95^{\circ} \mathrm{C}$ for $5 \mathrm{~s}, 60^{\circ} \mathrm{C}$ for $30 \mathrm{~s}$. After the reaction was completed, melting curve analysis was performed. The relative expression level of the target genes was calculated by the $2^{-\Delta \Delta C t}$ method [48]. The lncRNAs and target gene primers are shown in Supplemental Table S1.

\section{Statistical analyses}

All data are presented as the mean \pm SD. Student's $t$ tests were performed to compare means, and $P<0.05$ was considered statistically significant.

\section{Supplementary information}

Supplementary information accompanies this paper at https://doi.org/10. 1186/s12863-020-00850-6.

Additional file 1: Table S1. Differentially expressed mRNAs and IncRNAs between follicular phase and luteal phase in MM sheep.

Additional file 2: Table S2. Differentially expressed mRNAs and IncRNAs between follicular phase and luteal phase in WW sheep.

Additional file 3: Table S3. The differentially expressed mRNAs GO enrichment analyses between follicular phase and luteal phase in MM sheep.

Additional file 4: Table S4. The differentially expressed mRNAs GO enrichment analyses between follicular phase and luteal phase in WW sheep.

Additional file 5: Table S5. KEGG enrichment analysis of differentially expressed mRNAs between follicular phase and luteal phase in MM and WW sheep.

Additional file 6: Table S6. KEGG enrichment analysis of differentially expressed IncRNAs between follicular phase and luteal phase in MM and WW sheep.

Additional file 7 : Table S7. Details of primer sequences and expected product sizes of IncRNAs and IncRNA targets used for RT-qPCR.

\footnotetext{
Abbreviations

LncRNAs: Long noncoding RNAs; MAPK: The mitogen-activated protein kinase cascade; HPA: The hypothalamic-pituitary-adrenal axis; HPG: The hypothalamic-pituitary-gonadal axis; CRH: Corticotropin releasing hormone; ACTH: The release of adrenocorticotropic hormone; GnRH: Gonadotropin releasing hormone; CREB1: Cyclic AMP response element binding protein 1; BRAF: Serine/threonine kinase; mGCs: Mouse granulocytes;

NTRK2: Neurotrophic receptor tyrosine kinase 2; LHCGR: Luteinizing hormone receptor; FSH: Follicle stimulating hormone; SMAD1: Mothers against decapentaplegic homolog 1; BMP15: Bone morphogenetic protein 15; TGFBR1: TGF-1 receptor subtypes; TGFBR2: Type 2 receptor subtypes; MAS: Marker-assisted selection
}

\section{Acknowledgements}

We thank James Cummins, PhD, from EDANZ Group (www.edanzediting. com/ac) for editing a draft of this manuscript.

\section{Authors' contributions}

These studies were designed by QX and MXC, who performed the experimental analyses and prepared the figures and Tables. QX and QLL analyzed the data and drafted the manuscript. MXC contributed to revisions of the manuscript. XSZ, JLZ, XFG and MXC assisted in interpreting the results and revised the final version of the manuscript. All authors read and approved the final manuscript for publication.

\section{Funding}

This research was funded by the following bodies: National Natural Science Foundation of China (31472078 and 31772580); Earmarked Fund for China Agriculture Research System (CARS-38), Central Public-interest Scientific Institution Basal Research Fund (2018-YWF-YB-1, Y2017JC24, 2017ywf-zd-13); Agricultural Science and Technology Innovation Program of China (ASTIP-IAS13); China Agricultural Scientific Research Outstanding Talents and Their Innovative Teams Program, China High-level Talents Special Support Plan Scientific and Technological Innovation Leading Talents Program (W02020274); Joint Funds of The National Natural Science Foundation of China and The Government of Xinjiang Uygur Autonomous Region of China (U1130302); Tianjin Agricultural Science and Technology Achievements Transformation and Popularization Program (201704020); The Youth Innovative Research and Experimental Project of Tianjin Academy of Agricultural Sciences (201915). The APC was funded by the National Natural Science Foundation of China (31472078). The funding bodies had no role in the design of the study and collection, analysis, and interpretation of data and in writing the manuscript.

\section{Availability of data and materials}

The following information was supplied regarding data availability: Data is available at the Sequence Read Archive (SRA222893).

\section{Ethics approval and consent to participate}

All the Small-tailed Han sheep were euthanized and were authorized by the Science Research Department (in charge of animal welfare issue) of the Institute of Animal Sciences, Chinese Academy of Agricultural Sciences (IASCAAS; Beijing, China). In addition, ethical approval of animal survival was given by the animal ethics committee of IAS-CAAS (No. IAAS 2019-449, 18 September 2019).

Consent for publication

Not applicable.

\section{Competing interests}

The authors declare that they have no competing interests.

\section{Author details}

${ }^{1}$ Key Laboratory of Animal Genetics and Breeding and Reproduction of the Ministry of Agriculture and Rural Affairs, Institute of Animal Science, Chinese Academy of Agricultural Sciences, Beijing 100193, P. R. China. ${ }^{2}$ College of Life Sciences, Langfang Normal University, Langfang 065000, P.R. China. ${ }^{3}$ State Key Laboratory of Sheep Genetic Improvement and Healthy Production, Xinjiang Academy of Agricultural and Reclamation Sciences, Shihezi 832000, P. R. China. ${ }^{4}$ Tianjin Institute of Animal Sciences, Tianjin 300381, P. R. China.

Received: 22 September 2019 Accepted: 30 March 2020

Published online: 06 April 2020

References

1. Di R, Chu MX, Li YL, Zhang L, Fang L, Feng T, Cao GL, Chen HQ, Li XW. Predictive potential of microsatellite markers on heterosis off ecundity in crossbred sheep. Mol Biol Rep. 2012;39(3):2761-6.

2. Chu M, Jia L, Zhang Y, Jin M, Chen H, Fang L, Di R, Cao G, Feng T, Tang Q, Ma Y, Li K. Polymorphisms of coding region of BMPR-IB gene and their relationship with litter size in sheep. Mol Biol Rep. 2011:38(6):4071-6.

3. Miao X, Luo Q, Zhao H, Qin X. Co-expression analysis and identification of fecundity-related long non-coding RNAs in sheep ovaries. Sci Rep. 2016;6(1): 39398. 
4. Miao X, Luo Q, Zhao H, Qin X. An integrated analysis of miRNAs and methylated genes encoding mRNAs and IncRNAs in sheep breeds with different fecundity. Front Physiol. 2017:8:1049.

5. Yang H, Wang F, Li F, Ren C, Pang J, Wan Y, Wang Z, Feng X, Zhang $Y$. Comprehensive analysis of long non-coding RNA and mRNA expression patterns in sheep testicular maturation. Biol Reprod. 2018;99(3):650-61.

6. Zheng J, Wang Z, Yang H, Yao X, Yang P, Ren C, Wang F, Zhang Y. Pituitary transcriptomic study reveals the differential regulation of IncRNAs and mRNAs related to prolificacy in different FecB genotyping sheep. Genes (Basel). 2019. https://doi.org/10.3390/genes10020157.

7. Feng X, Li FZ, Wang F, Zhang GM, Pang J, Ren CF, Zhang TT, Yang H, Wang $Z Y$, Zhang YL. Genome-wide differential expression profiling of mRNAs and IncRNAs associated with prolificacy in Hu sheep. Biosci Rep. 2018. https:// doi.org/10.1042/BSR20171350.

8. Jimena P, Castilla JA, Peran F, Ramirez JP, Jr FV, Molina R, Vergara F, Herruzo A. Adrenal hormones in human follicular fluid. Acta Endocrinol, 1992;127(5):403.

9. Magiakou MA, Mastorakos G, Webster E, Chrousos GP. The hypothalamicpituitary-adrenal axis and the female reproductive system. Ann N Y Acad Sci. 1997;816(1):42-56.

10. Gao Y, Chen F, Kong QQ, Ning SF, Yuan HJ, Lian HY, Luo MJ, Tan JH. Stresses on female mice impair oocyte developmental potential: effects of stress severity and duration on oocytes at the growing follicle stage. Reprod Sci. 2016:23(9):1148-57.

11. Yuan HJ, Han X, Nan H, Guo LW, Shuai G, Juan L, Min G, Tian JH. Glucocorticoids impair oocyte developmental potential by triggering apoptosis of ovarian cells via activating the Fas system. Sci Rep. 2016;6: 24036.

12. Whirledge S, Cidlowski JA. Glucocorticoids and reproduction: traffic control on the road to reproduction. Trends Endocrinol Metab. 2017;28(6):399-415.

13. Bakhtiarizadeh MR, Hosseinpour B, Arefnzhad B, Shamabadi N, Salami SA. In silico prediction of long intergenic non-coding RNAs in sheep. Genome. 2016:59(4):263-75.

14. Zhou C, Zhang T, Liu F, Zhou J, Ni X, Huo R, Shi R. The differential expression of mRNAs and long noncoding RNAs between ectopic and eutopic endometria provides new insights into adenomyosis. Mol BioSyst. 2015;12(2):362-70.

15. Wang Y, Xue S, Liu X, Hu T, Qiu X, Zhang J, Lei M. Analyses of long noncoding RNA and mRNA profiling using RNA sequencing during the preimplantation phases in pig endometrium [J]. Sci Rep. 2016;6:20238.

16. Herbison AE, Robert P, Jean-Remi P, Mora JM, Hurst PR. Gonadotropinreleasing hormone neuron requirements for puberty, ovulation, and fertility. Endocrinology. 2008;149(2):597-604.

17. Lehman MN, Hileman SM, Goodman RL. Neuroanatomy of the kisspeptin signaling system in mammals: comparative and developmental aspects. Adv Exp Med Biol. 2015;784:27-62.

18. Rivier C, Vale W. Corticotropin-releasing factor (CRF) acts centrally to inhibit growth hormone secretion in the rat. Endocrinology. 1984;114(6):2409-11.

19. Gomes LG, Bachega TASS, Mendonca BB. Classic congenital adrenal hyperplasia and its impact on reproduction. Fertil and Steril. 2019;111(1):7-12.

20. Dobson H, Fergani C, Routly JE, Smith RF. Effects of stress on reproduction in ewes. Anim Reprod Sci. 2012;130(3-4):135-40.

21. Ciechanowska M, Lapot M, Antkowiak B, Mateusiak K, Paruszewska E, Malewski T, Paluch M, Przekop F. Effect of short-term and prolonged stress on the biosynthesis of gonadotropin-releasing hormone ( $\mathrm{GnRH})$ and $\mathrm{GnRH}$ receptor (GnRHR) in the hypothalamus and GnRHR in the pituitary of ewes during various physiological states. Anim Reprod Sci. 2016;174:65-72.

22. Clarke IJ, Bartolini D, Conductier G, Henry BA. Stress increases gonadotropin inhibitory hormone cell activity and input to gnRH cells in ewes. Endocrinology. 2016;157(11):en20161513.

23. Gubbay O, Rae MT, McNeilly AS, Donadeu FX, Zeleznik AJ, Hillier SG. CAMP response element-binding (CREB) signalling and ovarian surface epithelial cell survival. J Endocrinol. 2006;191(1):275-85.

24. Pei JH, Fujimoto $Y$, Yamauchi N, Hattori MA. Real-time monitoring of cAMP response element binding protein signaling in porcine granulosa cells modulated by ovarian factors. Mol Cell Biochem. 2006;290(1-2):177-84

25. Suman R, Androulla E, Zara J, Laura P, Maon HD. Metformin inhibits folliclestimulating hormone (FSH) action in human granulosa cells: relevance to polycystic ovary syndrome. J Clin Endocrinol Metab. 2013;98(9):E1491-500.

26. Natalie YO, Noa S, Naomi MB, Sarah E, Moriah K, Manna PR, Stocco DM, Joseph $\mathrm{O}$. Transcription of steroidogenic acute regulatory protein in the rodent ovary and placenta: alternative modes of cyclic adenosine 3', 5'-monophosphate dependent and independent regulation. Endocrinology. 2009:150(2):977-89.

27. Sirotkin AV, Gupta K, Kapoor R, Dwivedi A. MicroRNA-145 targets Smad1 in endometrial stromal cells and regulates decidualization in rat. J Mol Med (Berl). 2019;97(4):509-22

28. Guo M, Li Y, Chen Y, Guo X, Yuan Z, Jiang Y. Genome-wide mapping of estrogen receptor a binding sites by ChIP-seq to identify genes related to sexual maturity in hens. Gene. 2018;642:32-42.

29. LV X, Wang D, Ma Y, Long Z. Analysis of the oncogene BRAF mutation and the correlation of the expression of wild-type BRAF and CREB1 in endometriosis. Int J Mol Med. 2018;41(3):1349-56.

30. Zhang P, Wang J, Lang H, Wang W, Liu X, Liu H, Tan C, Li X, Zhao Y, Wu X. Knockdown of CREB1 promotes apoptosis and decreases estradiol synthesis in mouse granulosa cells. Biomed Pharmacother. 2018;105:1141-6.

31. Lei C, Liu K, Zhao Z, Blai HT, Peng Z, Li D, Ma RZ. Identification of sheep ovary genes potentially associated with off-season reproduction. J Genet Genomics. 2012:39(4):181-90.

32. Dorfman MD, Cecilia GR, Zefora A, Bredford K, Alejandro L, Dissen GA, Juan MC, David GG, Francisco G, Baoji X. Loss of Ntrk2/Kiss1r signaling in oocytes causes premature ovarian failure. Endocrinology. 2014;155(8):3098-111.

33. Kawai T, Richards JS, Shimada M. The cell type-specific expression of Lhcgr in mouse ovarian cells: evidence for a DNA-demethylation-dependent mechanism. Endocrinology. 2018;159(5):2062-74

34. Caterina C, Tripurani SK, Large MJ, Edson MA, Creighton CJ, Hawkins SM, Kovanci E, Kaartinen V, Lydon JP, DeMayo FJ, Matzuk MM. Activin-like kinase 2 functions in peri-implantation uterine signaling in mice and humans. PLoS Genet. 2013;9(11):e1003863.

35. Kim MR, Park DW, Lee JH, Choi DS, Hwang KJ, Ryu HS, Min CK. Progesterone-dependent release of transforming growth factor-beta1 from epithelial cells enhances the endometrial decidualization by turning on the Smad signalling in stromal cells. Mol Hum Reprod. 2005;11(11):801-8.

36. Moore RK, Otsuka F, Shimasaki S. Molecular basis of bone morphogeneric protein-15 signaling in gramulosa cells. J Biol Chem. 2003:278(1):304-10.

37. Claire G, Kemp CF, Knight PG. Bone morphogenetic protein (BMP) ligands and receptors in bovine ovarian follicle cells: actions of BMP-4, -6 and -7 on granulosa cells and differential modulation of Smad-1 phosphorylation by follistatin. Reproduction. 2004;127(2):239-54.

38. Knight PG, Glister C. TGF-beta superfamily members and ovarian follicle development. Reproduction. 2006;132(2):191-206.

39. Li XJ, Ye JW, Han X, Qiao RM, Li XL, Lv G, Wang KJ. Whole-genome sequencing identifies potential candidate genes for reproductive traits in pigs. Genomics. 2019. https://doi.org/10.1016/j.ygeno.2019.01.014.

40. Wang Q, Wang N, Cai R, Zhao F, Xiong Y, Li X, Wang A, Lin P, Jin Y. Genomewide analysis and functional prediction of long non-coding RNAs in mouse uterus during the implantation window. Oncotarget. 2017;8(48):84360-72.

41. Langmead B, Salzberg SL. Fast gapped-read alignment with bowtie 2. Nat Methods. 2012;9(4):357-9.

42. Pertea M, Kim D, Pertea GM, Leek JT, Salzerg SL. Transcript-level expression analysis of RNA-seq experiments with HISAT, StringTie and Ballgown. Nat Protoc. 2016;11(9):1650-67.

43. Trapnell C, Wiliams BA, Pertea G, Mortazavi A, Kwan G, Ven Baren MJ, Salzberg SL, Wold BJ, Pachter L. Transcript assembly and quantification by RNA-Seq reveals unannotated transcripts and isoform switching during cell differentiation. Nat Biotechnol. 2010;28(5):511-5.

44. Sun L, Luo H, Bu D, Zhao G, Yu K, Zhang C, Liu Y, Chen R, Zhao Y. Utilizing sequence intrinsic composition to classify protein-coding and long noncoding transcripts. Nucleic Acids Res. 2013:41(17):e166.

45. Kang YJ, Yang DC, Kong L, Hou M, Meng YQ, Wei L, Gao G. CPC2: a fast and accurate coding potential calculator based on sequence intrinsic features. Nucleic Acids Res. 2017;45(W1):W12-6.

46. Bateman A, Birney E, Cerruti L, Durbin R, Etwiller L, Eddy SR, Griffiths-Jones S, Howe $\mathrm{KL}$, Marshall M, Sonnhammer EL. The Pfam protein families database. Nucleic Acids Res. 2002;30(1):276-80.

47. Alessandro F, Irene B. Long non-coding RNAs: new players in cell differentiation and development. Nat Rev Genet. 2014;15(1):7-21.

48. Schmittgen TD, Libak KJ. Analyzing real-time PCR data by the comparative C(T) method. Nat Protoc. 2008;3(6):1101-8.

\section{Publisher's Note}

Springer Nature remains neutral with regard to jurisdictional claims in published maps and institutional affiliations. 